\title{
A Mean Value Formula for Elliptic Curves
}

\author{
Rongquan Feng ${ }^{1}$ and Hongfeng $\mathrm{Wu}^{2}$ \\ ${ }^{1}$ LMAM, School of Mathematical Sciences, Peking University, Beijing 100871, China \\ ${ }^{2}$ College of Science, North China University of Technology, Beijing 100144, China
}

Correspondence should be addressed to Hongfeng Wu; whfmath@gmail.com

Received 1 July 2014; Accepted 18 August 2014; Published 25 August 2014

Academic Editor: Jiyou Li

Copyright (C) 2014 R. Feng and H. Wu. This is an open access article distributed under the Creative Commons Attribution License, which permits unrestricted use, distribution, and reproduction in any medium, provided the original work is properly cited.

It is proved in this paper that, for any point on an elliptic curve, the mean value of $x$-coordinates of its $n$-division points is the same as its $x$-coordinate and that of $y$-coordinates of its $n$-division points is $n$ times that of its $y$-coordinate.

\section{Introduction}

Let $K$ be a field with $\operatorname{char}(K) \neq 2,3$ and let $\bar{K}$ be the algebraic closure of $K$. Every elliptic curve $E$ over $K$ can be written as a classical Weierstrass equation as follows:

$$
E: y^{2}=x^{3}+a x+b
$$

with coefficients $a, b \in K$. A point $Q$ on $E$ is said to be smooth (or nonsingular) if $\left(\partial f /\left.\partial x\right|_{Q}, \partial f /\left.\partial y\right|_{Q}\right) \neq(0,0)$, where $f(x, y)=y^{2}-x^{3}-a x-b$. The point multiplication is the operation of computing

$$
n P=\underbrace{P+P+\cdots+P}_{n}
$$

for any point $P \in E$ and a positive integer $n$. The multiplication-by- $n$ map

$$
\begin{aligned}
{[n]: E } & \longrightarrow E \\
P & \longmapsto n P
\end{aligned}
$$

is an isogeny of degree $n^{2}$. For a point $Q \in E$, any element of $[n]^{-1}(Q)$ is called an $n$-division point of $Q$. Assume that $(\operatorname{char}(K), n)=1$. In this paper, the following result on the mean value of the $x, y$-coordinates of all the $n$-division points of any smooth point on an elliptic curve is proved.

Theorem 1. Let $E$ be an elliptic curve defined over $K$ and let $Q=\left(x_{Q}, y_{Q}\right) \in E$ be a point with $Q \neq \mathcal{O}$. Set

$$
\Lambda=\left\{P=\left(x_{P}, y_{P}\right) \in E(\bar{K}) \mid n P=Q\right\} .
$$

Then

$$
\begin{aligned}
& \frac{1}{n^{2}} \sum_{P \in \Lambda} x_{P}=x_{\mathrm{Q}}, \\
& \frac{1}{n^{2}} \sum_{P \in \Lambda} y_{P}=n y_{\mathrm{Q}} .
\end{aligned}
$$

According to Theorem 1, let $P_{i}=\left(x_{i}, y_{i}\right), i=1,2, \ldots, n^{2}$, be all the points such that $n P=Q$ and let $\lambda_{i}$ be the slope of the line through $P_{i}$ and $Q$; then $y_{\mathrm{Q}}=\lambda_{i}\left(x_{\mathrm{Q}}-x_{i}\right)+y_{i}$. Therefore,

$$
n^{2} y_{Q}=\sum_{i=1}^{n^{2}} \lambda_{i} \cdot \frac{\sum_{i=1}^{n^{2}} x_{i}}{n^{2}}-\sum_{i=1}^{n^{2}} \lambda_{i} x_{i}+\sum_{i=1}^{n^{2}} y_{i}
$$

Thus we have

$$
\begin{aligned}
y_{Q} & =\frac{\sum_{i=1}^{n^{2}} \lambda_{i}}{n^{2}} \cdot \frac{\sum_{i=1}^{n^{2}} x_{i}}{n^{2}}-\frac{\sum_{i=1}^{n^{2}} \lambda_{i} x_{i}}{n^{2}}+\frac{\sum_{i=1}^{n^{2}} y_{i}}{n^{2}} \\
& =\overline{\lambda_{i}} \cdot \overline{x_{i}}-\overline{\lambda_{i} x_{i}}+\overline{y_{i}},
\end{aligned}
$$

where $\overline{\lambda_{i}}, \overline{x_{i}}, \overline{\lambda_{i} x_{i}}$, and $\overline{y_{i}}$ are the average values of the variables $\lambda_{i}, x_{i}, \lambda_{i} x_{i}$, and $y_{i}$, respectively. Therefore,

$$
\mathrm{Q}=\left(x_{\mathrm{Q}}, y_{\mathrm{Q}}\right)=\left(\overline{x_{i}}, \overline{\lambda_{i}} \cdot \overline{x_{i}}-\overline{\lambda_{i} x_{i}}+\overline{y_{i}}\right)=\left(\overline{x_{i}}, \frac{1}{n} \overline{y_{i}}\right) .
$$

Remark 2. The discrete logarithm problem in elliptic curve $E$ is to find $n$ by given $P, Q \in E$ with $Q=n P$. The above theorem gives some information on the integer $n$. 


\section{Proof of Theorem 1}

To prove Theorem 1, define division polynomials [1] $\psi_{n} \in$ $\mathbb{Z}[x, y, a, b]$ on an elliptic curve $E: y^{2}=x^{3}+a x+b$ inductively as follows:

$$
\begin{gathered}
\psi_{0}=0, \\
\psi_{1}=1, \\
\psi_{2}=2 y \\
\psi_{3}=3 x^{4}+6 a x^{2}+12 b x-a^{2}, \\
\psi_{4}=4 y\left(x^{6}+5 a x^{4}+20 b x^{3}-5 a^{2} x^{2}-4 a b x-8 b^{2}-a^{3}\right), \\
\psi_{2 n+1}=\psi_{n+2} \psi_{n}^{3}-\psi_{n-1} \psi_{n+1}^{3}, \quad \text { for } n \geq 2, \\
2 y \psi_{2 n}=\psi_{n}\left(\psi_{n+2} \psi_{n-1}^{2}-\psi_{n-2} \psi_{n+1}^{2}\right), \quad \text { for } n \geq 3 .
\end{gathered}
$$

It can be checked easily by induction that the $\psi_{2 n}$ 's are polynomials. Moreover, $\psi_{n} \in \mathbb{Z}\left[x, y^{2}, a, b\right]$ when $n$ is odd, and $(2 y)^{-1} \psi_{n} \in \mathbb{Z}\left[x, y^{2}, a, b\right]$ when $n$ is even. Define the polynomial

$$
\phi_{n}=x \psi_{n}^{2}-\psi_{n-1} \psi_{n+1}
$$

for $n \geq 1$. Then $\phi_{n} \in \mathbb{Z}\left[x, y^{2}, a, b\right]$. Since $y^{2}=x^{3}+a x+b$, replacing $y^{2}$ by $x^{3}+a x+b$, one has $\phi_{n} \in \mathbb{Z}[x, a, b]$. So we can denote it by $\phi_{n}(x)$. Note that $\psi_{n} \psi_{m} \in \mathbb{Z}[x, a, b]$ if $n$ and $m$ have the same parity. Furthermore, the division polynomials $\psi_{n}$ have the following properties.

Lemma 3. Consider

$$
\psi_{n}=n x^{\left(n^{2}-1\right) / 2}+\frac{n\left(n^{2}-1\right)\left(n^{2}+6\right)}{60} a x^{\left(n^{2}-5\right) / 2}
$$

+ lower degree terms,

when $n$ is odd and

$$
\begin{gathered}
\psi_{n}=n y\left(x^{\left(n^{2}-4\right) / 2}+\frac{\left(n^{2}-1\right)\left(n^{2}+6\right)-30}{60} a x^{\left(n^{2}-8\right) / 2}\right. \\
+ \text { lower degree terms }),
\end{gathered}
$$

when $n$ is even.

Proof. We prove the result by induction on $n$. It is true for $n<5$. Assume that it holds for all $\psi_{m}$ with $m<n$. We give the proof only for the case for odd $n \geq 5$. The case for even $n$ can be proved similarly. Now let $n=2 k+1$ be odd, where $k \geq 2$. If $k$ is even, then by induction

$$
\begin{aligned}
& \psi_{k}=k y\left(x^{\left(k^{2}-4\right) / 2}+\frac{\left(k^{2}-1\right)\left(k^{2}+6\right)-30}{60}\right. \\
& \left.\quad \times a x^{\left(k^{2}-8\right) / 2}+\cdots\right) \\
& \psi_{k+2}=(k+2) y \\
& \times\left(x^{\left(k^{2}+4 k\right) / 2}+\frac{\left(k^{2}+4 k+3\right)\left(k^{2}+4 k+10\right)-30}{60}\right. \\
& \left.\quad \times a x^{\left(k^{2}+4 k-4\right) / 2}+\cdots\right)
\end{aligned}
$$

$$
\begin{aligned}
\psi_{k-1}= & (k-1) x^{\left(k^{2}-2 k\right) / 2} \\
& +\frac{(k-1)\left(k^{2}-2 k\right)\left(k^{2}-2 k+7\right)}{60} \\
& \times a x^{\left(k^{2}-2 k-4\right) / 2}+\cdots, \\
\psi_{k+1}= & (k+1) x^{\left(k^{2}+2 k\right) / 2} \\
& +\frac{(k+1)\left(k^{2}+2 k\right)\left(k^{2}+2 k+7\right)}{60} \\
& \times a x^{\left(k^{2}+2 k-4\right) / 2}+\cdots .
\end{aligned}
$$

Substituting $y^{4}$ by $\left(x^{3}+a x+b\right)^{2}$, we have

$$
\begin{aligned}
\psi_{k+2} \psi_{k}^{3}= & k^{3}(k+2) \\
& \times\left(x^{2 k^{2}+2 k}+\frac{4(k+1)\left(k^{3}+k^{2}+10 k+3\right)}{60}\right. \\
& \left.\times a x^{2 k^{2}+2 k-2}+\cdots\right), \\
\psi_{k-1} \psi_{k+1}^{3}= & (k-1)(k+1)^{3} x^{2 k^{2}+2 k} \\
& +\frac{4 k(k-1)\left(k^{3}+2 k^{2}+11 k+7\right)(k+1)^{3}}{60} \\
& \times a x^{2 k^{2}+2 k-2}+\cdots \cdot
\end{aligned}
$$


Therefore,

$$
\begin{aligned}
\psi_{2 k+1}= & \psi_{k+2} \psi_{k}^{3}-\psi_{k-1} \psi_{k+1}^{3} \\
= & (2 k+1) x^{2 k^{2}+2 k} \\
& +\frac{(2 k+1)\left(4 k^{2}+4 k\right)\left(4 k^{2}+4 k+7\right)}{60} \\
& \times a x^{2 k^{2}+2 k-2}+\cdots \\
= & (2 k+1) x^{\left((2 k+1)^{2}-1\right) / 2} \\
& +\frac{(2 k+1)\left((2 k+1)^{2}-1\right)\left((2 k+1)^{2}+6\right)}{60} \\
& \times a x^{\left((2 k+1)^{2}-5\right) / 2}+\cdots .
\end{aligned}
$$

The case when $k$ is odd can be proved similarly.

The following corollary follows immediately from Lemma 3.

\section{Corollary 4. Consider}

$$
\begin{gathered}
\psi_{n}^{2}=n^{2} x^{n^{2}-1}-\frac{n^{2}\left(n^{2}-1\right)\left(n^{2}+6\right)}{30} a x^{n^{2}-3}+\cdots, \\
\phi_{n}=x^{n^{2}}-\frac{n^{2}\left(n^{2}-1\right)}{6} a x^{n^{2}-2}+\cdots
\end{gathered}
$$

Proof of Theorem 1. Define $\omega_{n}$ as

$$
4 y \omega_{n}=\psi_{n+2} \psi_{n-1}^{2}-\psi_{n-2} \psi_{n+1}^{2} .
$$

Then for any $P=\left(x_{P}, y_{P}\right) \in E$, we have ([1])

$$
n P=\left(\frac{\phi_{n}\left(x_{P}\right)}{\psi_{n}^{2}\left(x_{P}\right)}, \frac{\omega_{n}\left(x_{P}, y_{P}\right)}{\psi_{n}\left(x_{P}, y_{P}\right)^{3}}\right) .
$$

If $n P=Q$, then $\phi_{n}\left(x_{P}\right)-x_{Q} \psi_{n}^{2}\left(x_{P}\right)=0$. Therefore, for any $P \in \Lambda$, the $x$-coordinate of $P$ satisfies the equation $\phi_{n}(x)-$ $x_{Q} \psi_{n}^{2}(x)=0$. From Corollary 4 , we have that

$$
\begin{aligned}
\phi_{n}(x)- & x_{\mathrm{Q}} \psi_{n}^{2}(x) \\
& =x^{n^{2}}-n^{2} x_{\mathrm{Q}} x^{n^{2}-1}+\text { lower degree terms. }
\end{aligned}
$$

Since $\sharp \Lambda=n^{2}$, every root of $\phi_{n}(x)-x_{Q} \psi_{n}^{2}(x)$ is the $x$ coordinate of some $P \in \Lambda$. Therefore,

$$
\sum_{P \in \Lambda} x_{P}=n^{2} x_{Q}
$$

by Vitae's theorem.

Now we prove the mean value formula for $y$-coordinates. Let $K$ be the complex number field $\mathbb{C}$ first and let $\omega_{1}$ and $\omega_{2}$ be complex numbers which are linearly independent over $\mathbb{R}$. Define the lattice

$$
L=\mathbb{Z} \omega_{1}+\mathbb{Z} \omega_{2}=\left\{n_{1} \omega_{1}+n_{2} \omega_{2} \mid n_{1}, n_{2} \in \mathbb{Z}\right\}
$$

and the Weierstrass $\wp$-function by

$$
\wp(z)=\wp(z, L)=\frac{1}{z^{2}}+\sum_{\omega \in L, \omega \neq 0}\left(\frac{1}{(z-\omega)^{2}}-\frac{1}{\omega^{2}}\right) .
$$

For integers $k \geq 3$, define the Eisenstein series $G_{k}$ by

$$
G_{k}=G_{k}(L)=\sum_{\omega \in L, \omega \neq 0} \omega^{-k} .
$$

Set $g_{2}=60 G_{4}$ and $g_{3}=140 G_{6}$; then

$$
\wp^{\prime}(z)^{2}=4 \wp(z)^{3}-g_{2} \wp(z)-g_{3} .
$$

Let $E$ be the elliptic curve given by $y^{2}=4 x^{3}-g_{2} x-g_{3}$. Then the map

$$
\begin{aligned}
& \frac{\mathbb{C}}{L} \longrightarrow E(\mathbb{C}) \\
& z \longmapsto\left(\wp(z), \wp^{\prime}(z)\right), \\
& 0 \longmapsto \infty
\end{aligned}
$$

is an isomorphism of groups $\mathbb{C} / L$ and $E(\mathbb{C})$. Conversely, it is well known [1] that, for any elliptic curve $E$ over $\mathbb{C}$ defined by $y^{2}=x^{3}+a x+b$, there is a lattice $L$ such that $g_{2}(L)=-4 a, g_{3}(L)=-4 b$ and there is an isomorphism between groups $\mathbb{C} / L$ and $E(\mathbb{C})$ given by $z \mapsto$ $\left(\wp(z),(1 / 2) \wp^{\prime}(z)\right)$ and $0 \mapsto \infty$. Therefore, for any point $(x, y) \in E(\mathbb{C})$, we have $(x, y)=\left(\wp(z),(1 / 2) \wp^{\prime}(z)\right)$ and $n(x, y)=\left(\wp(n z),(1 / 2) \wp^{\prime}(n z)\right)$ for some $z \in \mathbb{C}$.

Let $Q=\left(\wp\left(z_{\mathrm{Q}}\right),(1 / 2) \wp^{\prime}\left(z_{\mathrm{Q}}\right)\right)$ for a $z_{\mathrm{Q}} \in \mathbb{C}$. Then for any $P_{i} \in \Lambda, 1 \leq i \leq n^{2}$, there exist integers $j, k$ with $0 \leq j, k \leq n-1$, such that

$$
P_{i}=\left(\wp\left(\frac{z_{\mathrm{Q}}}{n}+\frac{j}{n} \omega_{1}+\frac{k}{n} \omega_{2}\right), \frac{1}{2} \wp^{\prime}\left(\frac{z_{\mathrm{Q}}}{n}+\frac{j}{n} \omega_{1}+\frac{k}{n} \omega_{2}\right)\right) .
$$

Thus,

$$
\sum_{j, k=0}^{n-1} \wp\left(\frac{z_{Q}}{n}+\frac{j}{n} \omega_{1}+\frac{k}{n} \omega_{2}\right)=n^{2} \wp\left(z_{Q}\right)
$$

which comes from $\sum_{i=1}^{n^{2}} x_{i}=n^{2} x_{\mathrm{Q}}$. Differentiate with respect to $z_{\mathrm{Q}}$, we have

$$
\sum_{j, k=0}^{n-1} \wp^{\prime}\left(\frac{z_{\mathrm{Q}}}{n}+\frac{j}{n} \omega_{1}+\frac{k}{n} \omega_{2}\right)=n^{3} \wp^{\prime}\left(z_{\mathrm{Q}}\right) .
$$

That is,

$$
\sum_{i=1}^{n^{2}} y_{i}=n^{3} y_{\mathrm{Q}}
$$

Secondly, let $K$ be a field of characteristic 0 and let $E$ be the elliptic curve over $K$ given by the equation $y^{2}=x^{3}+a x+b$. Then all of the equations describing the group law are defined 
over $\mathbb{Q}(a, b)$. Since $\mathbb{C}$ is algebraically closed and has infinite transcendence degree over $\mathbb{Q}, \mathbb{Q}(a, b)$ can be considered as a subfield of $\mathbb{C}$. Therefore we can regard $E$ as an elliptic curve defined over $\mathbb{C}$. Thus the result follows.

At last assume that $K$ is a field of characteristic $p$. Then the elliptic curve can be viewed as one defined over some finite field $\mathbb{F}_{q}$, where $q=p^{m}$ for some integer $m$. Without loss of generality, let $K=\mathbb{F}_{q}$ for convenience. Let $K^{\prime}=\mathbb{Q}_{q}$ be an unramified extension of the $p$-adic numbers $\mathbb{Q}_{p}$ of degree $m$, and let $\bar{E}$ be an elliptic curve over $K^{\prime}$ which is a lift of $E$. Since $(n, p)=1$, the natural reduction map $\bar{E}[n] \rightarrow E[n]$ is an isomorphism. Now for any point $Q \in E$ with $Q \neq \mathcal{O}$, we have a point $\bar{Q} \in \bar{E}$ such that the reduction point is $Q$. For any point $P_{i} \in E(\bar{K})$ with $n P_{i}=Q$, its lifted point $\bar{P}_{i}$ satisfies $n \bar{P}_{i}=\bar{Q}$ and $\bar{P}_{i} \neq \bar{P}_{j}$ whenever $P_{i} \neq P_{j}$. Thus,

$$
\sum_{i=1}^{n^{2}} y\left(\bar{P}_{i}\right)=n^{3} y(\bar{Q})
$$

since $K^{\prime}$ is a field of characteristic 0 . Therefore the formula $\sum_{i=1}^{n^{2}} y_{i}=n^{3} y_{\mathrm{Q}}$ holds by the reduction from $\bar{E}$ to $E$.

Remark 5.

(1) The result for $x$-coordinate of Theorem 1 holds also for the elliptic curve defined by the general Weierstrass equation $y^{2}+a_{1} x y+a_{3} y=x^{3}+a_{2} x^{2}+a_{4} x+a_{6}$.

(2) The mean value formula for $x$-coordinates was given in the first version of this paper [2] with a slightly complicated proof. The formula for $y$-coordinates was conjectured by Feng and Wu based on [2] and numerical examples in a personal email communication with Moody (June 1, 2010).

(3) Recently, some mean value formulae for twisted Edwards curves $[3,4]$ and other alternate models of elliptic curves were given by $[5,6]$.

\section{An Application}

Let $E$ be an elliptic curve over $K$ given by the Weierstrass equation $y^{2}=x^{3}+a x+b$. Then we have a nonzero invariant differential $\omega=d x / y$. Let $\phi \in \operatorname{End}(E)$ be a nonzero endomorphism. Then $\phi^{*} \omega=\omega \circ \phi=c_{\phi} \omega$ for some $c_{\phi} \in$ $\bar{K}(E)$, since the space $\Omega_{E}$ of differential forms on $E$ is a 1 dimensional $\bar{K}(E)$-vector space. Since $c_{\phi} \neq 0$ and $\operatorname{div}(\omega)=0$, we have

$$
\operatorname{div}\left(c_{\phi}\right)=\operatorname{div}\left(\phi^{*} \omega\right)-\operatorname{div}(\omega)=\phi^{*} \operatorname{div}(\omega)-\operatorname{div}(\omega)=0
$$

Hence $c_{\phi}$ has neither zeros nor poles and $c_{\phi} \in \bar{K}$. Let $\varphi$ and $\psi$ be two nonzero endomorphisms; then

$$
\begin{aligned}
c_{\varphi+\psi} \omega & =(\varphi+\psi)^{*} \omega=\varphi^{*} \omega+\psi^{*} \omega=c_{\varphi} \omega+c_{\psi} \omega \\
& =\left(c_{\varphi}+c_{\psi}\right) \omega .
\end{aligned}
$$

Therefore, $c_{\varphi+\psi}=c_{\varphi}+c_{\psi}$. For any nonzero endomorphism $\phi$, set $\phi(x, y)=\left(R_{\phi}(x), y S_{\phi}(x)\right)$, where $R_{\phi}$ and $S_{\phi}$ are rational functions. Then

$$
c_{\phi}=\frac{R_{\phi}^{\prime}(x)}{S_{\phi}(x)}
$$

where $R_{\phi}^{\prime}(x)$ is the differential of $R_{\phi}(x)$. In particular, for any positive integer $n$, the map $[n]$ on $E$ is an endomorphism. Set $[n](x, y)=\left(R_{n}(x), y S_{n}(x)\right)$. From $c_{[1]}=1$ and $[n]=[1]+$ $[(n-1)]$, we have

$$
c_{[n]}=\frac{R_{n}^{\prime}(x)}{S_{n}(x)}=n .
$$

For any $Q=\left(x_{Q}, y_{Q}\right) \in E$ and any

$$
P=\left(x_{P}, y_{P}\right) \in \Lambda=\left\{P=\left(x_{P}, y_{P}\right) \in E(\bar{K}) \mid n P=Q\right\},
$$

we have $y_{P}=y_{\mathrm{Q}} / S_{n}\left(x_{P}\right)$. Therefore, Theorem 1 gives

$$
\sum_{P \in \Lambda} \frac{1}{S_{n}\left(x_{P}\right)}=\sum_{P \in \Lambda} \frac{y_{P}}{y_{Q}}=\frac{1}{y_{Q}} \sum_{P \in \Lambda} y_{P}=n^{3} .
$$

Thus,

$$
\begin{aligned}
& \sum_{P \in \Lambda} \frac{1}{R_{n}^{\prime}\left(x_{P}\right)}=\sum_{P \in \Lambda} \frac{1}{n \cdot S_{n}\left(x_{P}\right)}=\frac{1}{n} \sum_{P \in \Lambda} \frac{1}{S_{n}\left(x_{P}\right)}=n^{2}, \\
& \sum_{P \in \Lambda} \frac{x_{Q}}{R_{n}^{\prime}\left(x_{P}\right)}=x_{Q} \sum_{P \in \Lambda} \frac{1}{R_{n}^{\prime}\left(x_{P}\right)}=n^{2} x_{\mathrm{Q}}=\sum_{P \in \Lambda} x_{P} .
\end{aligned}
$$

\section{Conflict of Interests}

The authors declare that there is no conflict of interests regarding the publication of this paper.

\section{Acknowledgments}

This work was supported by the National Natural Science Foundation of China (no. 11101002, no. 61370187, and no. 11271129) and Beijing Natural Science Foundation (no. 1132009).

\section{References}

[1] J. H. Silverman, The Arithmetic of Elliptic Curves, vol. 106 of Graduate Texts in Mathematics, Springer, New York, NY, USA, 1986.

[2] R. Feng and H. Wu, "A mean value formula for elliptic curves,” Cryptology ePrint Archive Report 2009/586, 2009, http://eprint.iacr.org/.

[3] D. Bernstein, P. Birkner, M. Joye, T. Lange, and C. Peters, "Twisted Edwards curves," in Progress in CryptologyAFRICACRYPT 2008, vol. 5023 of Lecture Notes in Computer Science, pp. 389-405, Springer, Berlin, Germany, 2008.

[4] H. M. Edwards, "A normal form for elliptic curves," Bulletin of the American Mathematical Society, vol. 44, no. 3, pp. 393-422, 2007. 
[5] D. Moody, "Mean value formulas for twisted Edwards curves," 2010, http://eprint.iacr.org/2010/142.pdf.

[6] D. Moody, Divison Polynomials for Alternate Models of Elliptic Curves, http://eprint.iacr.org/2010/630.pdf. 


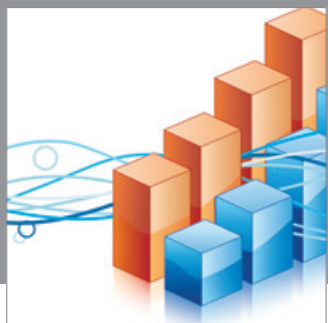

Advances in

Operations Research

mansans

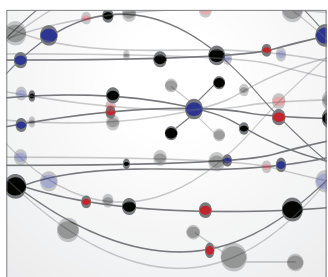

The Scientific World Journal
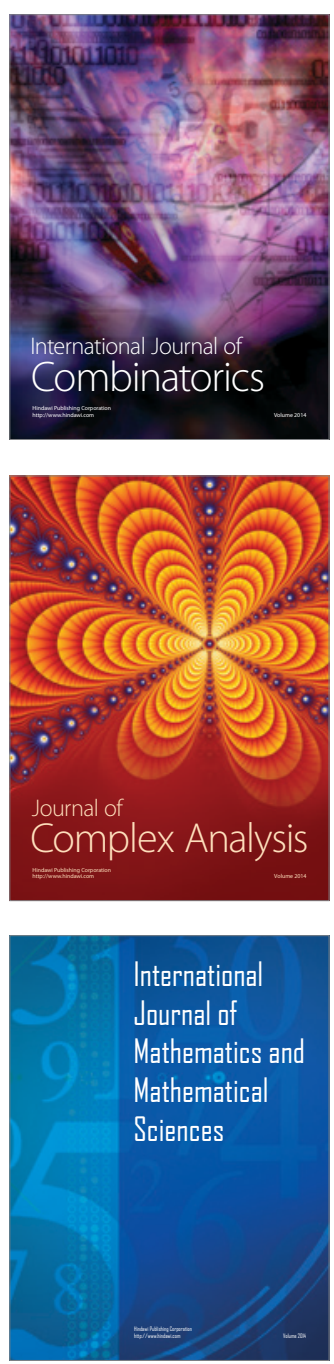
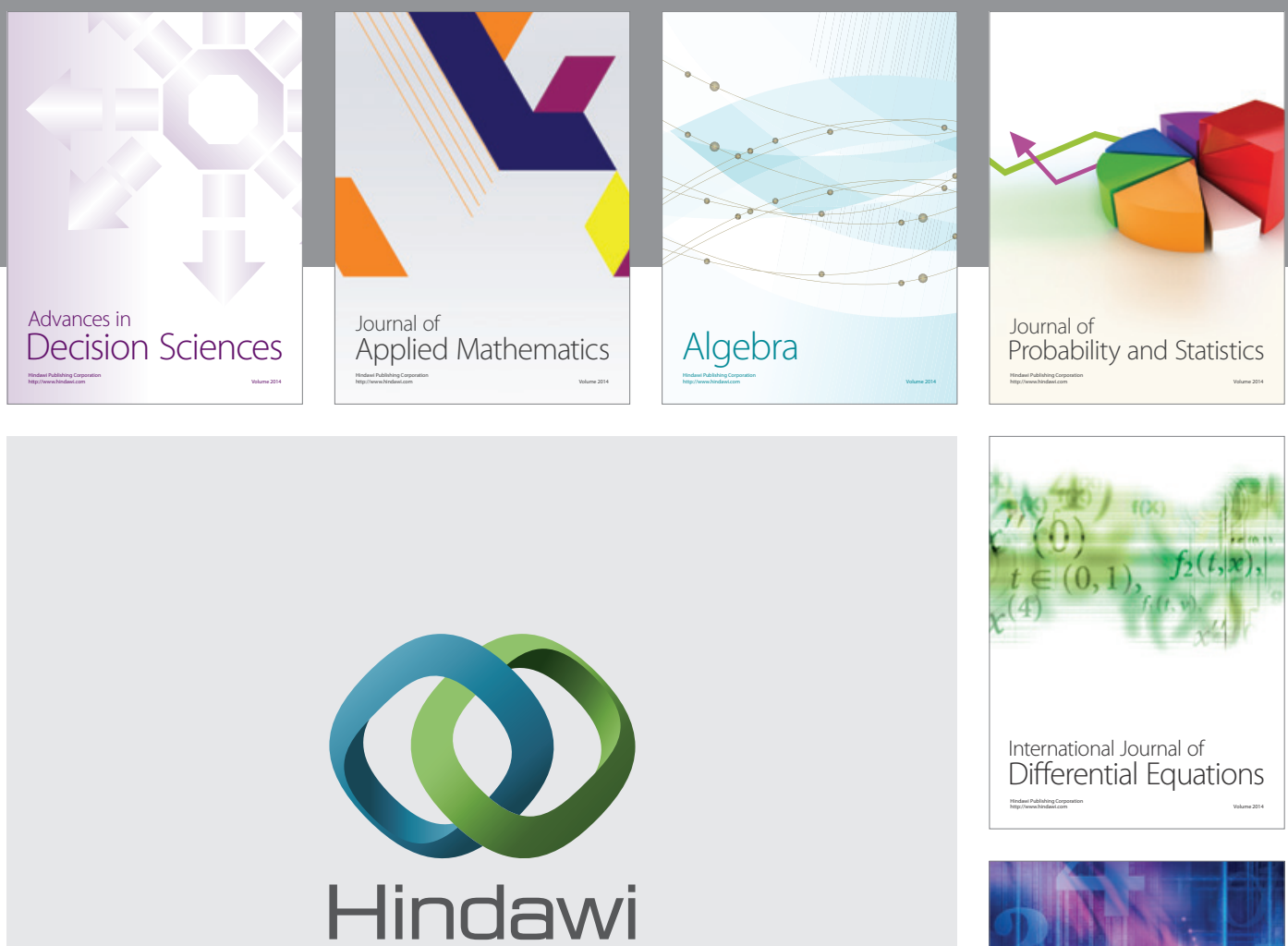

Submit your manuscripts at http://www.hindawi.com
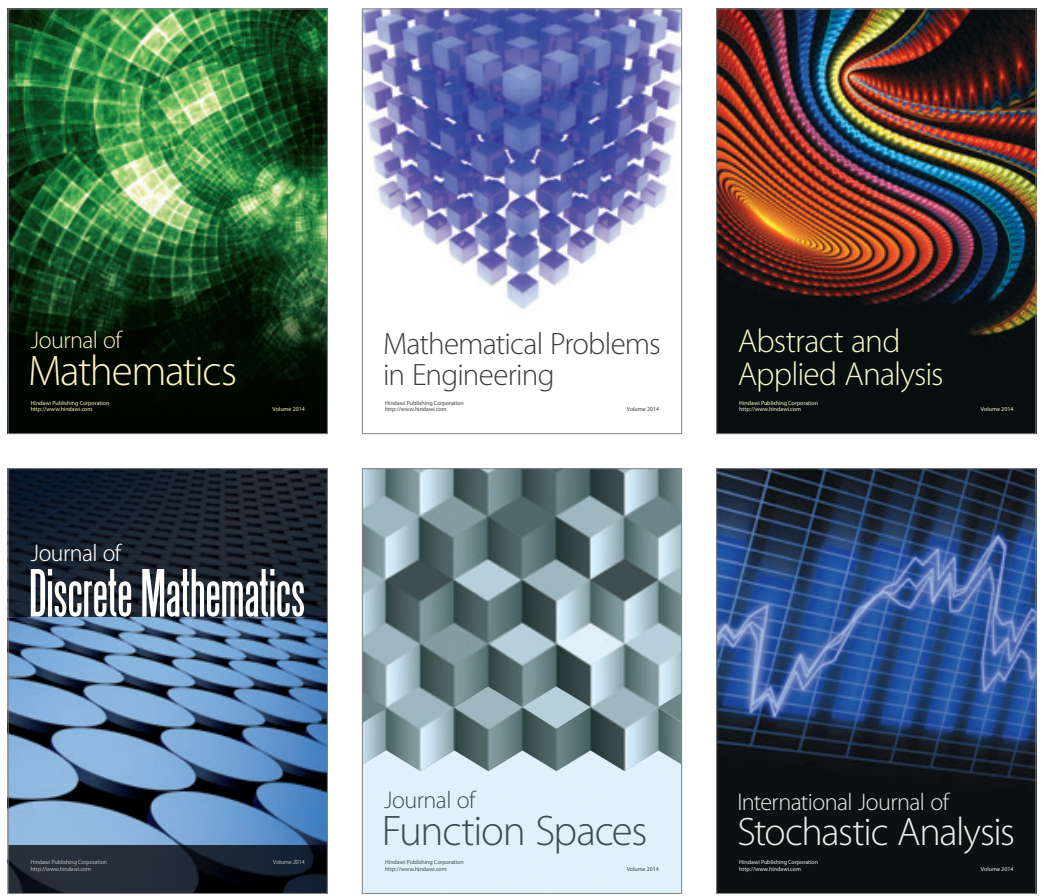

Journal of

Function Spaces

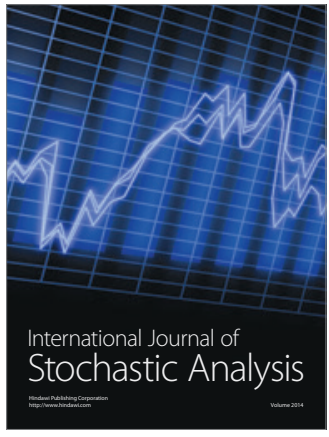

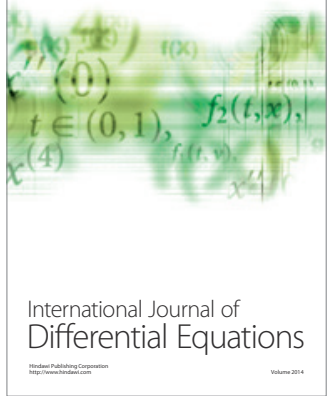
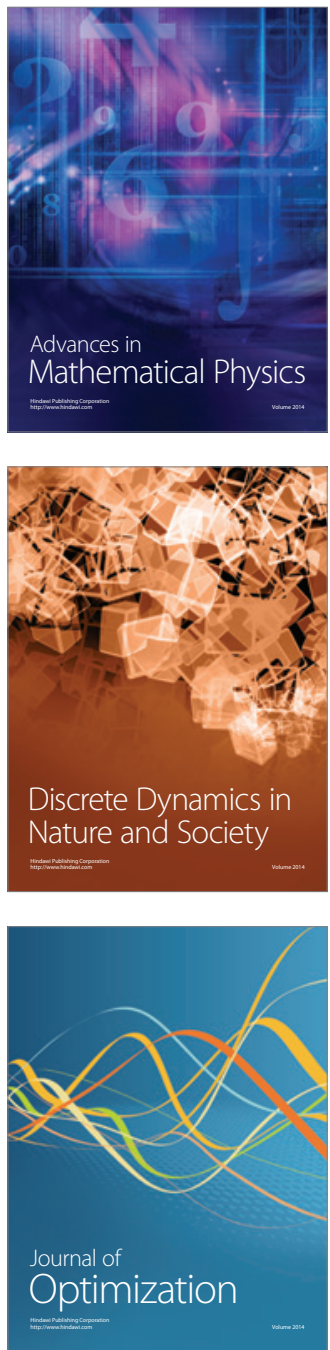